\title{
Analysis of hemocoagulation tests for prediction of venom- -induced consumption coagulopathy development after Viperidae bite
}

\author{
Valenta J, Stach Z, Porizka M, Michalek P \\ Department of Anaesthesiology, Resuscitation and Intensive Care, First Faculty of Medicine, Charles University \\ and General University Hospital, Prague, Czech Republic. pavel.michalek@vfn.cz
}

\begin{abstract}
OBJECTIVE: Viperidae snakes are responsible for $95 \%$ of the bites caused by exotic-bred snakes in our country. Their envenoming may be associated with a severe acute coagulation disorder - venom-induced consumption coagulopathy (VICC). Thus, its early prediction is vital for an adequate therapy including antivenom delivery. MATERIAL AND METHODS: Laboratory coagulation tests of 14 patients suffering from VICC were processed and statistically analyzed to evaluate the importance of individual parameters in the time after the bite. RESULTS: The pathological values of D-dimer (D-dim) and fibrinogen (FBG) were found to be the first indicators of VICC development, with a median time of 4.55 hours since the bite, while median values for prothrombin time and international normalized ratio (PT/INR), activated partial thromboplastin time (APTT), and thrombin time (TT) were $5.9 \mathrm{~h}, 8.15 \mathrm{~h}$, and $5.5 \mathrm{~h}$, respectively. In the first samples, the values of D-dim were found to be pathologically increased in all 14 patients, while pathological levels of FBG were seen only in 11 cases. PT/ INR and APTT were prolonged in 8 and 6 cases, respectively.

CONCLUSION: An increase in D-dim values was found to be the first parameter signaling developing VICC in all analyzed cases (Tab. 2, Ref. 12). Text in PDF www.elis.sk.

KEY WORDS: snakebite, Viperidae, envenoming, hemocoagulation, venom-induced consumption coagulopathy.
\end{abstract}

\section{Introduction}

Venom of most American, African, and Asian Viperidae snakes (subfamily Viperinae and Crotalinae) cause inter alia a hemocoagulation disorder, namely venom-induced consumption coagulopathy (VICC) (1-2). These snakes are popular among breeders in our country (3). During the period from January 1998 to -October 2018, 95 of the 111 bites by exotic snakes were inflicted by Viperidae snakes, and 19 of them suffered from VICC.

Only few of the essential antivenoms are readily available at the national toxinology center (TC). As per algorithms of national TC, an envenomed patient is transported to a specialized center after initial examination if envenoming is present. If antivenom is indicated (any systemic manifestations including apparent derangement of plasmatic coagulation system (4), rare thrombotic microangiopathy (5-6), or eventual severe local infliction), the antivenom is provided and administered.

Department of Anaesthesiology, Resuscitation and Intensive Care, First Faculty of Medicine, Charles University and General University Hospital, Prague, Czech Republic.

Address for correspondence: P. Michalek, MD, PhD, DESA, MSc, Department of Anaesthesia and Intensive Medicine, 1st Medical Faculty, Charles University and General University Hospital, U nemocnice 2, CZ-120 21 Prague, Czech Republic. Phone: +420.224962243, Fax: +420.224962118 Supported by MZCZ-DRO-VFN64165
Therefore, in such cases it becomes necessary to import antivenom urgently from foreign toxinology holding centers. Thus, diagnosis of imminent VICC must be performed as soon as possible and potential severity of the disorder should be appropriately estimated.

The aim of this retrospective study was to analyze the predictive value of individual laboratory coagulation parameters for fast and valid estimation of potential VICC development. Concurrently, the onset of harmful peak values of laboratory coagulation parameters of ongoing VICC during treatment was monitored. Diagnosis of VICC was confirmed when FBG levels decreased, D-dim levels were elevated, and coagulation times were prolonged in consecutive laboratory tests.

\section{Material and methods}

The laboratory data of the basic cohort of 95 victims from TC database who suffered exotic Viperidae family snakebites were retrospectively processed. The Ethical Committee of the hospital reviewed and consented to the protocol (391/18 S-IV). Exclusion criteria for this cohort were: dry bite (14 cases), bites from European vipers not causing VICC (17 cases), isolated nonserious local infliction of bite only and other symptoms not affecting coagulation (39 cases), a single finding of D-dim up to 2000 $\mu \mathrm{g} / \mathrm{L}$ without any other or further pathologies (6 cases), previous VICC patients with incomplete laboratory data (4 cases), and 1 
patient coming in on the 4th day after the bite with already ongoing VICC.

The final cohort included 14 males, with a mean age of 39 years (16-67 years). In the study group, VICC was caused by the following snakes: Atheris nitschei, Atheris squamigera, Bothrops asper, Calloselasma rhodostoma, Cerastes cerastes, Crotalus adamanteus, Crotalus durissus, Deinagkistrodon acutus, Echis carinatus (2 cases), Echis pyramidum, Macrovipera schweizeri, Proatheris superciliaris, Trimeresurus (Cryptelytrops) albolabris.

The results of prothrombin time and international normalized ratio (PT/INR), activated partial thromboplastin time (APTT), thrombin time (TT), fibrinogen (FBG) Clauss assay, D-dim (immunochemical assay), antithrombin III percentage (AT), and platelets count (PLT) were evaluated using statistical methods. First pathological values, peak values, and the duration until their development were recorded and statistically analyzed.

\section{Statistics}

Time and levels of the first pathological values and peak values of measured parameters were extracted from the Excel tables. The number of pathological values was assessed in the first laboratory sample of coagulation parameters. Furthermore, total number of pathological versus normal values during the entire course of VICC was evaluated.

This data set was subsequently analyzed using GraphPad Instat 3 (GraphPad Software, La Jolla, CA, USA) statistical program. All data obtained was initially checked for normal distribution using Shapiro-Wilk test. Normally distributed data was presented as mean and $95 \%$ confidence interval (CI) while data not normally distributed as median and 25-75\% inter-quartile range (IQR). The categorical data was then analyzed using two-tailed Fisher's exact test and numerical data was compared using the WilcoxonMann-Whitney $U$ test. All $p$ values $<0.05$ were considered as statistically significant.
Tab. 2. Number of pathological values in the first sample and number of cases reaching pathological values during VICC.

\begin{tabular}{lcc}
\hline \multirow{2}{*}{$\mathrm{n}=14$} & \multicolumn{2}{c}{ Number of pathologies } \\
\cline { 2 - 3 } & In 1st examination & In VICC course \\
\hline INR N $\leq 1.2$ & $8(57.1 \%)$ & $14(100 \%)$ \\
APTT N $=20-40 \mathrm{~s}$ & $6(42.9 \%)$ & $10(71.4 \%)$ \\
TT N $=12-18 \mathrm{~s}$ & $9(64.3 \%)$ & $14(100 \%)$ \\
FBG N $=2-4 \mathrm{gL}^{-1}$ & $11(78.6 \%)$ & $14(100 \%)$ \\
AT N $\geq 75 \%$ & $3(21.40 \%)$ & $6(42.9 \%)$ \\
D-dim N $\leq 190 \mu \mathrm{gL}^{-1}$ & $14(100 \%)$ & $14(100 \%)$ \\
PLT N $\geq 150 * 10^{\circ} \mathrm{L}^{-1}$ & $5(35.7 \%)$ & $8(57.1 \%)$ \\
\hline
\end{tabular}

$\mathrm{N}$ - normal values

\section{Results}

The medians, absolute ranges, and interquartile ranges of the first laboratory coagulation pathologies between the bite and their occurrence are shown in Table 1.

The pathological values of D-dim and FBG were found to be the first findings signaling development of VICC; with a median time of 4.55 hours (h) since the bite, while median times for INR, APTT, and TT were $5.9 \mathrm{~h}, 8.15 \mathrm{~h}$ and $5.5 \mathrm{~h}$, respectively. The values of AT and PLT remained mostly within normal range in the first sample, and the median times for development of pathology were $12.75 \mathrm{~h}$ and $18.65 \mathrm{~h}$, respectively.

An analysis of the first sample after the bite showed that Ddim was found pathologically elevated in 14 cases (100\%); FBG in 11 cases only $(78.6 \%)$. In the three remaining cases, values of FBG vs D-dim were: $2.4 \mathrm{~g} \mathrm{~L}^{-1}$ vs $484 \mu \mathrm{g} \mathrm{L}^{-1} ; 2 \mathrm{~g} \mathrm{~L}^{-1}$ vs $640 \mu \mathrm{g} \mathrm{L}^{-1}$; $2.19 \mathrm{~g} \mathrm{~L}^{-1} \mathrm{vs}>6400 \mu \mathrm{g} \mathrm{L}^{-1}$. The ratios of pathological vs normal values of INR, APTT, and TT in the first coagulation tests after bite were significantly lower (Tab. 2).

Differences between pathological values of parameters found in the first examination were statistically significant in D-dim vs INR $(p=0.016)$, D-dim vs APTT $(p=0.002)$, and D-dim vs TT

Tab. 1. Values of the first pathological and peak results in the time since the snakebite.

\begin{tabular}{|c|c|c|c|c|c|c|}
\hline \multirow{2}{*}{$\mathrm{n}=14$} & \multicolumn{3}{|c|}{ Pathology in first examination } & \multicolumn{3}{|c|}{ Peak of values } \\
\hline & median & range & IQR & median & range & IQR \\
\hline$\overline{\mathrm{INR} N} \leq 1.2$ & 1.755 & $1.24-10$ & $1.38-10$ & 2.65 & $1.3-10$ & $1.5-10$ \\
\hline Time (h) & 5.9 & $1.5-21$ & $4.6-18$ & 9.5 & $1.5-28$ & $4.6-18$ \\
\hline APTT N $=20-40 \mathrm{~s}$ & 127.8 & $36.8-180$ & $39-180$ & 127.8 & $36.8-180$ & $39-180$ \\
\hline Time (h) & 8.15 & $1.5-21$ & $4.6-16.5$ & 10.65 & $1.5-28$ & $4.6-18$ \\
\hline $\mathrm{TT} \mathrm{N}=12-18 \mathrm{~s}$ & 43.15 & $19.1-180$ & $22.8-180$ & 180 & $19.1-180$ & $28.1-180$ \\
\hline Time (h) & 5.55 & $1.5-27$ & $4.25-10.8$ & 9.5 & $1.5-28$ & $4.6-18$ \\
\hline Time (h) & 4.55 & $1.5-21$ & $2.05-7.3$ & 11.25 & $1.5-28$ & $5.0-18$ \\
\hline AT $N \geq 75 \%$ & 78.5 & $62-93$ & $71-88$ & 78.5 & $57-93$ & $62-88$ \\
\hline Time (h) & 12.75 & $5.5-27$ & $9.0-21$ & 21.25 & $5.5-52$ & $10.5-24$ \\
\hline $\mathrm{D}-\operatorname{dim} \mathrm{N} \leq 190 \mu \mathrm{gL}^{-1}$ & 4413.5 & $210-6400$ & $605-6400$ & 6400 & $781-6500$ & $4348-6400$ \\
\hline Time (h) & 4.55 & $0.7-21$ & $1.5-5.6$ & 9.5 & $1.5-28$ & $4.5-16.5$ \\
\hline PLT $\mathrm{N} \geq 150^{*} 10^{9} \mathrm{~L}^{-1}$ & 131 & $22-253$ & $93-173$ & 116 & $16-234$ & $22-173$ \\
\hline Time $(\bar{h})$ & 18.65 & $1.5-63$ & $5.5-24.5$ & 32 & 4-110 & $16.5-49$ \\
\hline
\end{tabular}

Numbers closest to the pathological values are included if no pathological finding in any parameter during VICC was recorded. For laboratory limit INR $>10$ is included 10 ; similar APTT $>180=180 ;$ TT $>180=180 ; \mathrm{FBG}<0.1=0.1 ; \mathrm{D} \operatorname{dim}>6400=6400$. Time $(\mathrm{h})-$ time after the snakebite. $\mathrm{N}-$ normal value. 
$(p=0.041)$, whereas this was not observed between FBG and INR, APTT, TT ( $p=0.420,0.120,0.678)$, as well as between FBG and D-dim $(p=0.222)$.

The peaks of individual coagulation parameters in the time since the bite are shown in Table 1 but have been modified by antivenom therapy. FBG values reached clinically dangerous levels - median $0.2 \mathrm{~g} \mathrm{~L}^{-1}$ in $11.25 \mathrm{~h}$ (in three cases $<0.1 \mathrm{~g} \mathrm{~L}^{-1}$ in $5 \mathrm{~h}, 4.5$ $\mathrm{h}$, and $1.5 \mathrm{~h}$, respectively). TT was found as the most sensitive clotting time test indicating VICC severity (median $180 \mathrm{~s}$ in 9.5 $\mathrm{h}$ ); while the INR median time of $2.65 \mathrm{~h}$ did not reach an alarming value. Moreover, APTT results were found within the normal range in four of the cases despite ongoing VICC with hypofibrinogenemia and consumption.

\section{Discussion}

In case of a developing or already developed VICC, clinical symptoms of coagulation disorder may not be present, even in the case of severe hypofibrinogenemia with impending life-threatening bleeding (7). Diagnosis and estimation of the severity depend on the laboratory examinations carried out shortly after the bite and the interpretation of its results.

Some studies recommend the PT value to be sufficient as a diagnostic marker due to its easy availability and low cost (8). This parameter may be acceptable in a developed VICC and is adequate for diagnostics in areas where snakes are native, especially when antivenoms are immediately available and possibilities of laboratory tests are limited.

In areas beyond the native occurrence of snakes that cause VICC, envenoming of breeders is only sporadic, and the availability of antivenoms can be problematic. Early and correct findings will prevent treatment delays by confirming the need of urgent import of antivenom and would prevent unnecessary antiserum delivery in cases that it is not required as suggested by the tests (9). For these reasons, it is crucial to diagnose emerging VICC early and with high probability.

The results of this analysis confirm our hypothesis: a high suspicion of VICC should be made based on a slight decrease in FBG levels and especially on a rise in D-dim levels soon after the bite, regardless of the finding of normal PT and APTT values in some cases $(8,10)$.

We recommend considering the abnormal values of D-dim as the first sign of emerging VICC. FBG levels oscillate even in healthy population between $2-4 \mathrm{~g} \mathrm{~L}^{-1}$, and a slight decrease may remain unregistered $(8,11)$. On the contrary, D-dim levels above normal value, i.e. $>190 \mu \mathrm{g} \mathrm{L}^{-1}$ mostly indicate a pathological status. This situation was found in 3 of the cases in our cohort. Even a slight, isolated elevation of D-dim soon after the bite can most likely signal further development of VICC.

\section{Conclusion}

The most sensitive parameters for suspicion of VICC development were: pathological value of D-dim found in all cases and apparent decrease of FBG levels in $76.8 \%$ of impending VICC in the first laboratory sample. For analysis of the duration from the bite to VICC development, the scattering of values was too variable in this small cohort to be statistically significant, however median time for abnormal values of D-dim and FBG were $4.55 \mathrm{~h}$ and life-threatening FBG decrease was $11.25 \mathrm{~h}$. In the first samples, the values of PT/INR and APTT were found to be normal or mostly showed a moderate increase, and therefore do not exclude impending VICC. PLT count and AT activity mostly did not reflect other changes in coagulation parameters. The results of this study confirm an elevation in D-dim levels soon after the bite to be the first marker of impending VICC. Targeted antivenom therapy based on the elevations in D-dim may become an important point in intensive care of the envenomed patient (12).

\section{References}

1. Slagboom J, Kool J, Harrison RA, Casewell NR. Haemotoxic snake venoms: their functional activity, impact on snakebite victims and pharmaceutical promise. Br J Haematol 2017; 177 (6): 947-959.

2. White J. Snake venoms and coagulopathy. Toxicon 2005; 45 (8): 951967.

3. Valenta J, Stach Z, Michalek P. Exotic snake bites in the Czech republic - epidemiological and clinical aspects during 15-year period. Clin Toxicol (Phila) 2014; 52 (4): 258-264.

4. Babaie M, Salmanizadeh H, Zolfagharian H, Alizadeh H. Properties of biological and biochemical effects of the Iranian saw-scaled viper (Echis carinatum) venom. Bratisl Med J 2014, 115 (7): 434-438.

5. Ireland G, Brown SG, Buckley NA et al. Changes in serial laboratory test results in snakebite patients: when can we safely exclude envenoming? Med J Aust 2010; 193 (5): 285-290.

6. Gerardo CJ,Vissoci JR, Brown MW, Bush SP. Coagulation parameters in copperhead compared to other Crotalinae envenomation: secondary analysis of the $\mathrm{F}\left(\mathrm{ab}^{\prime}\right)$, versus Fab antivenom trial. Clin Toxicol 2017; 55 (2): 109-114.

7. Maduwage K, Isbister GK. Current treatment for venom-induced consumption coagulopathy resulting from snakebite. PLoS Negl Trop Dis. 2014; 10: e3220.

8. Berling I, Isbister GK. Hematologic effects and complications of snake envenoming. Transfus Med Rev 2015; 29 (2): 82-89.

9. Isbister GK, Maduwage K, Shahmy S et al. Diagnostic 20-min whole blood clotting test in Russell's viper envenoming delays antivenom administration. Q J Med 2013; 106 (10): 925-932.

10. Olives TD, M. Topeff JM, Willhite LA, Kubic VL, Keyler DE, Cole JB. Complete clinical course of envenomation by Protobothrops mangshanensis: delayed coagulopathy and response to Trimeresurus albolabris antivenom. Clin Toxicol (Phila) 2016; 54 (3): 290-292.

11. Li QB, Huang GW, Kinjoh K, Nakamura M, Kosugi T. Hematological studies on DIC-like findings observed in patients with snakebite in south China. Toxicon 2001; 39 (7): 943-948.

12. Valenta J, Stach Z, Michalek P. Severe snakebite envenoming in intensive care. Prague Med Rep 2016; 117 (4): 153-163.

Received April 27, 2019.

Accepted May 31, 2019. 